\title{
Direct incorporation of the NKT-cell activator a-galactosylceramide into a recombinant Listeria monocytogenes improves breast cancer vaccine efficacy
}

\author{
M Singh ${ }^{1}$, W Quispe-Tintaya ${ }^{1}$, D Chandra ${ }^{1}$, A Jahangir ${ }^{1}, \mathrm{M} \mathrm{M} \mathrm{Venkataswamy}{ }^{1,3}, \mathrm{~T} \mathrm{~W} \mathrm{Ng}^{1}$, S Sharma-Kharkwal ${ }^{1}$, \\ L J Carreño ${ }^{1,2}$, S A Porcelli*,1 and C Gravekamp*,1 \\ ${ }^{1}$ Department of Microbiology and Immunology, Albert Einstein College of Medicine, 1300 Morris Park Avenue, Bronx, NY 10461, \\ USA and ${ }^{2}$ Millennium Institute on Immunology and Immunotherapy, Facultad de Medicina, Universidad de Chile, Avenue \\ Independencia \#1027, Santiago 8380453, Chile
}

Background: Immune suppression in the tumour microenvironment remains a major limitation to successful immunotherapy of cancer. In the current study, we analysed whether the natural killer T cell-activating glycolipid $\alpha$-galactosylceramide could overcome immune suppression and improve vaccination against metastatic breast cancer.

Methods: Mice with metastatic breast cancer (4T1 model) were therapeutically treated with a Listeria monocytogenes-based vaccine expressing tumour-associated antigen Mage-b followed by $\alpha$-galactosylceramide as separate agents, or as a complex of $\alpha$-galactosylceramide stably incorporated into Listeria-Mage-b. Effects on metastases, tumour weight, toxicity and immune responses were determined.

Results: Sequential treatments of mice with established 4T1 breast carcinomas using Listeria-Mage-b followed by $\alpha$-galactosylceramide as a separate agent was highly effective at reducing metastases, but was accompanied by severe liver toxicity. In contrast, combined therapy using Listeria-Mage-b modified by incorporation of $\alpha$-galactosylceramide resulted in nearly complete elimination of metastases without toxicity. This was associated with a significant increase in the percentage of natural killer T cells in the spleen, and an increase in natural killer cell activity and in T cell responses to Mage-b.

Conclusions: Our results suggest that direct incorporation of $\alpha$-galactosylceramide into a live bacterial vaccine vector is a promising non-toxic new approach for the treatment of metastatic breast cancer.

Breast cancer is the most common cancer among women worldwide, and $\sim 30 \%$ of cases progress to metastatic disease, which is difficult or impossible to treat effectively (Youlden et al, 2012). Current treatment options for metastatic cancer include surgery followed by chemotherapy or radiation, or other adjuvant therapy (Cardoso et al, 2012). Despite aggressive treatment, elimination of metastases or residual tumour cells after initial treatment is often incomplete, and removal of residual disease by chemotherapy is prevented by chemoresistance (Gonzalez-Angulo et al, 2007). This underlines the urgent need for new effective therapies against metastatic disease.

It has been shown in mice and humans that vaccines can have a favourable effect on metastases (Niethammer et al, 2002; Marchand et al, 2003; Kruit et al, 2005; Kim et al, 2008) but that vaccine efficacy is strongly reduced by immune suppression in the tumour microenvironment (TME; Gajewski et al, 2006). In a *Correspondence: Professor C Gravekamp; E-mail: claudia.gravekamp@einstein.yu.edu or Professor SA Porcelli; E-mail: steven.porcelli@einstein.yu.edu
${ }^{3}$ Current address: National Institute of Mental Health and Neuroscience (NIMHANS), Bangalore, India. 
previous study, we developed a vaccine based on a non-pathogenic strain of Listeria monocytogenes (LM) expressing the tumourassociated antigen (TAA) Mage-b (LM-Mb; Kim et al, 2008). Listeria monocytogenes is an intracellular bacterium that has the capacity to deliver antigens through infection into antigenpresenting cells (APCs) such as dendritic cells (DCs), monocytes and macrophages with high efficiency (Paterson and Maciag, 2005). We have also demonstrated that LM infects tumour cells, which can lead to cytolytic effects through a mechanism involving induction of high levels of reactive oxygen species, and sensitises the infected tumour cells for recognition by LM-specific CD8 ${ }^{+} \mathrm{T}$ cells (Kim et al, 2009). When administered prior to tumour establishment in an aggressive mouse model of metastatic breast cancer (4T1), LM-Mb treatment resulted in strong CD8 ${ }^{+}$T-cell responses to both Mage-b and LM and an almost complete elimination of metastatic disease (Kim et al, 2008). However, when administered in a therapeutic setting (i.e., after establishment of primary $4 \mathrm{~T} 1$ tumours), LM-Mb treatment was only moderately effective against metastatic breast cancer, and induced relatively weak $\mathrm{CD}^{+}{ }^{+} \mathrm{T}$-cell responses to Mage-b, due to strong immune suppression in the TME (Kim et al, 2009).

To overcome the immune suppression that is characteristic of tumour-bearing hosts, immunologic adjuvants that can promote robust immune responses and augment the effects of therapeutic vaccines are greatly needed. Glycolipids of the $\alpha$-galactosylceramide family $(\alpha \mathrm{GC})$ represent one potentially useful class of adjuvants that have shown promise in preclinical studies for immunotherapy of cancers (Metelitsa, 2011; Yamasaki et al, 2011). These glycolipids mediate their effects on the immune system by binding to an MHC class I-like molecule called CD1d, creating a complex that is recognised by a population of conserved effector lymphocytes known as natural killer $\mathrm{T}$ (NKT) cells (Bendelac et al, 2007). Formation of intracellular complexes of CD1d with $\alpha \mathrm{GC}$ in APCs initiates rapid NKT-cell activation (Bendelac et al, 2007), resulting in the production of Th1-associated cytokines such as IFN $\gamma$ and IL-12p70, maturation of $\mathrm{CD} 8 \alpha^{+}$DCs in the lymph nodes and subsequent activation of NK and conventional T cells (Hermans et al, 2003; Venkataswamy et al, 2009). This cascade of immune reactions that is initiated by NKT cells in response to $\alpha$ GC has been shown in mouse models to generate innate and adaptive immunity against a wide range of cancers and infections (Brigl et al, 2003; Behar and Porcelli, 2007; Bendelac et al, 2007).

Based on these observations we hypothesised that addition of $\alpha \mathrm{GC}$ to $\mathrm{LM}-\mathrm{Mb}$ vaccine could improve therapeutic efficacy, in part through enhancement of specific T cell responses to Mage-b. When these two agents were used as combination therapy in the 4T1 model, $\alpha \mathrm{GC}$ significantly improved the therapeutic vaccine efficacy of $\mathrm{LM}-\mathrm{Mb}$ as demonstrated by the almost complete elimination of the metastases. However, the administration of these two agents sequentially caused severe and in some cases fatal toxicity to the liver, leading us to explore other novel strategies. Drawing on previous experience using direct incorporation of relatively low doses of $\alpha \mathrm{GC}$ into live Mycobacterium bovis BCG to improve vaccine efficacy (Venkataswamy et al, 2009), we developed a similar approach for direct incorporation of the glycolipid into live LM organisms. This generated a vaccine that was equally effective against metastatic breast cancer compared to sequential administration of LM-Mb and $\alpha \mathrm{GC}$ as separate agents, but without any apparent toxicity. The powerful anti-metastatic effect of vaccination with LM-Mb modified by direct incorporation of $\alpha \mathrm{GC}$ correlated with an increase in the frequency of splenic NKT cells, and improved responses of Mage-b-specific $\mathrm{CD}^{+} \mathrm{T}$ and $\mathrm{NK}$ cells. This approach may provide a basis for new strategies to safely improve vaccine efficacy against metastatic cancer through augmentation of multiple innate and adaptive immune mechanisms.

\section{MATERIALS AND METHODS}

Mice. Normal female BALB/c mice aged 3 months were obtained from Charles River Laboratories and maintained in the animal facility of Albert Einstein College of Medicine according to the guidelines of the Association for Assessment and Accreditation of Laboratory Animal Care, and according the guidelines of the Albert Einstein Institute for Animal Studies. All mice were kept under biosafety level 2 conditions, as required for Listeria monocytogenes.

Cells, cell culture and plasmids. The $4 \mathrm{~T} 1$ cell line, derived from a spontaneous mammary carcinoma in a $\mathrm{BALB} / \mathrm{c}$ mouse (Aslakson and Miller, 1992), was cultured in Dulbecco's Modified Eagle's Medium supplemented with $10 \%$ fetal bovine serum, $1 \mathrm{~mm}$ mixed non-essential amino acids, $2 \mathrm{~mm}$ L-glutamine, insulin (0.5 USP units $\left.\mathrm{ml}^{-1}\right)$, penicillin (100 units $\mathrm{ml}^{-1}$ ) and streptomycin $\left(100 \mu \mathrm{g} \mathrm{ml}^{-1}\right)$. The DNA plasmids pcDNA3.1-Mage-b (Sypniewska et al, 2005), pcDNA3.1-LacZ (Invitrogen, Grand Island, NY, USA) and pCMV-GM-CSF (Chambers and Johnston, 2003) were purified using the Endo-Free Mega kit from Qiagen (Valencia, CA, USA).

LM-based vaccine. The LM-Mb strain was developed in an earlier study (Kim et al, 2008). This was constructed in the prfA-negative XFL-7 strain (referred to as LM in this study), which lacks the positive regulatory factor A that is a central mediator of virulence (Singh et al, 2005). The vaccine strain was transformed with LM plasmid pGG-34, which encodes prfA and amino acids 311-660 of murine Mage-b fused to a non-cytolytic form of listeriolysin $\mathrm{O}$ (Gunn et al, 2001; Kim et al, 2008). Complementation of prfA expression by the plasmid does not fully restore virulence, but enforces retention of the plasmid during infection (Gunn et al, 2001; Singh et al, 2005).

Incorporation of $\alpha \mathrm{GC}$ into live LM-Mb. The $\alpha \mathrm{GC}$ used in this study ((2S, 3S, 4R)-1-O-( $\alpha$-D-galactopyranosyl)- $N$-hexacosanoyl2-amino-1,3,4-octadecanetriol), also known in previous studies as

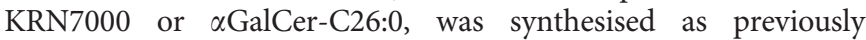
described ( $\mathrm{Yu}$ et al, 2005), and stored as solvent-free aliquots in glass vials at $-20^{\circ} \mathrm{C}$. The glycolipid was reconstituted either in $100 \%$ dimethylsulphoxide (DMSO) at $100 \mu \mathrm{M}$ for in vitro studies, or in aqueous vehicle consisting of PBS with $0.05 \%$ Tween 20 (Life Technologies, Grand Island, NY, USA) and 0.1\% DMSO at $500 \mu \mathrm{M}$ for in vivo studies. The incorporation of $\alpha \mathrm{GC}$ into live $\mathrm{LM}$ was performed using a method similar to that described previously for M. bovis BCG (Venkataswamy et al, 2009). Briefly, $\alpha \mathrm{GC}$ was solubilised at a concentration of $2.3 \mu \mathrm{m}$ in glass vials by addition of warm $\left(37^{\circ} \mathrm{C}\right)$ brain-heart infusion medium containing $5 \%$ tyloxapol, followed by sonication for $5 \mathrm{~min}$, heating at $80^{\circ} \mathrm{C}$ for $2 \mathrm{~min}$ and vortexing for $1 \mathrm{~min}$. Then, $450 \mu \mathrm{l}$ of the $2.3 \mu \mathrm{M}$ glycolipid solution was immediately diluted into $50 \mathrm{ml}$ of warm BHI to give the required final concentrations of $20 \mathrm{~nm}$ glycolipid and $0.05 \%$ tyloxapol, and this was inoculated with $500 \mu \mathrm{l}$ of a LM-Mb mid-log phase culture $\left(\mathrm{OD}_{600} 0.5-0.8\right)$. Following incubation for $4-6 \mathrm{~h}$ at $37^{\circ} \mathrm{C}$ to reach mid-log phase $\left(\mathrm{OD}_{600} 0.5-1.0\right)$, the bacterial culture was then immediately aliquoted in $1 \mathrm{ml}$ vials and frozen at $-80^{\circ} \mathrm{C}$. For subsequent use, the bacteria were thawed, harvested by centrifugation, washed three times with saline and resuspended in saline for injection. In order to determine the incorporation of $\alpha \mathrm{GC}$ into LM, we measured the ability of the bacteria to activate an iNKT-cell hybridoma as described in Supplementary Figure S1.

Tumour challenge and immunotherapy regimens. Mice were challenged with $10^{4} 4 \mathrm{~T} 1$ cells injected into the mammary fat pad as described previously (Kim et al, 2008), and then treated using therapeutic regimens to compare the effects of combined $\mathrm{LM}-\mathrm{Mb}$ and $\alpha \mathrm{GC}$ treatment either as separate agents or as 
glycolipid-modified bacteria. Initial pilot studies showed that when these two agents were administered separately, anti-metastatic effects were greater when $\mathrm{LM}-\mathrm{Mb}$ treatment was given prior to a series of injections of $\alpha \mathrm{GC}$, as opposed to the reverse order. Moreover, when LM-Mb and $\alpha \mathrm{GC}$ were administered at the same time points, the anti-metastatic effect was less compared to LM-Mb and $\alpha \mathrm{GC}$ administered sequentially, and animals developed severe liver toxicity (data not shown). Based on these observations we administered LM-Mb first followed by $\alpha \mathrm{GC}$ in all subsequent experiments when used as separate agents. The first protocol consisted of three therapeutic immunisations with LM-Mb followed by three injections of $\alpha \mathrm{GC}$. Mice received $10^{4} 4 \mathrm{~T} 1$ tumour cells in the mammary fat pad on day 0 , followed by three i.p. injections of LM-Mb or LM $\left(10^{4} \mathrm{CFU}\right)$, or saline on days 3, 6 and 9, and then three i.p. injections of $\alpha \mathrm{GC}$ ( $100 \mathrm{ng}$ in $200 \mu \mathrm{l}$ vehicle) i.p. on days 14, 15 and 16. The second protocol consisted of five therapeutic immunisations with LM-Mb modified by direct incorporation of $\alpha \mathrm{GC}$ (I- $\alpha \mathrm{GC}-\mathrm{LM}-\mathrm{Mb})$. Mice received $10^{4} 4 \mathrm{~T} 1$ tumour cells in the mammary fat pad on day 0 , followed by i.p. injections of $10^{4}$ CFU of I- $\alpha$ GC-LM-Mb on days 3, 6, 9, 12 and 15 . All mice were euthanised for the analysis of metastatic disease and immunology on day 18. The total number of metastases per mouse was determined as previously described (Kim et al, 2008). For studies carried out to determine effects on survival, mice were maintained until they succumbed spontaneously, or were terminated upon appearance of severe pre-morbid symptoms requiring euthanasia as specified by our approved animal use protocol.

Activation of iNKT cells in vivo. BALB/c mice were injected i.p. with the inert vehicle (PBS plus $0.05 \%$ Tween 20 and $0.1 \%$ DMSO), $10^{4}$ CFU LM, $10^{4}$ CFU I- $\alpha$ GC-LM or 4 nmoles of free $\alpha$ GC. Sera were assayed at the indicated times for IL- 4 , and IFN $\gamma$ by capture ELISA as previously described (Yu et al, 2005). Alternatively, $\mathrm{BALB} / \mathrm{c}$ mice receiving $4 \mathrm{~T} 1$ tumour cells and therapeutic immunisations with LM-Mb or I- $\alpha$ GC-LM-Mb (see above) were injected with 4 nmoles of free $\alpha \mathrm{GC} 15$ days after the injection of tumour cells. After the indicated times, splenocyte single-cell suspensions were obtained and stained with anti-mouse TCR-FITC (clone H57-597, BD Biosciences, San Jose, CA, USA) and $\alpha \mathrm{GC}$ loaded mouse CD1d tetramers-APC, prepared as previously described (Venkataswamy et al, 2009). Samples were acquired using a LSR II Flow Cytometer (BD Biosciences) and analysed using FlowJo software (TreeStar Software, Ashland, OR, USA).

Flow cytometry analyses. Cells were isolated from blood as described previously (Kim et al, 2008; Castro et al, 2009). Briefly, red blood cells were lysed according standard protocols, and the remaining leukocyte population was used for analysis. Cells were first incubated with Golgi-Plug for $6 \mathrm{~h}$ (BD Biosciences), then with an Fc blocker (mAb 2.4G2, anti-CD16/CD32), and finally with specific fluorochrome-conjugated antibodies for the identification of different cell types. Anti-CD8 $\alpha-\mathrm{PE}$ (clone 53-6.7) antibodies were used to identify CD8 T cells, and anti-CD45-APC antibody (clone 30-F11) to identify the leucocyte population in tumour cell suspensions. To detect the production of intracellular cytokines, cell suspensions were processed using the BD Cytofix/Cytoperm kit (BD Biosciences) according to the manufacturer's instructions, and stained using antibodies to IFN $\gamma$ (clone XMG1.2). Appropriate isotype controls were used for each sample. All antibodies were purchased from BD Biosciences. For most samples, data of $1.5 \times 10^{5}$ and $3 \times 10^{5}$ cells were acquired using a FACSCalibur flow cytometer (BD Biosciences). However, in some cases where noted with samples derived from small tumours, limited cell numbers allowed acquisition of only $10^{4}$ cells. Cell debris and dead cells were excluded from the analyses based on forward and side scatter signals, and used Fixable Blue or Green Live/Dead Cell Stain kit (Invitrogen). All data analyses were done using FlowJo software (TreeStar).
ELISPOT. Spleen cells were isolated from vaccinated and control mice with 4T1 tumours and analysed for T cell responses to Mageb- and NK-cell responses to LM by ELISPOT as described previously (Kim et al, 2008; Chandra et al, 2013). To detect Mageb-specific immune responses, $4 \times 10^{5}$ spleen cells of vaccinated or control mice were transfected with plasmids pcDNA3.1-Mage-b and pCMV-GM-CSF ( $1 \mu \mathrm{g}$ of each plasmid per $5 \times 10^{6}$ spleen cells) using Lipofectamine 2000 (Life Technologies), as described previously (Kim et al, 2008). Expression of Mage-b by the transfected spleen cells was verified by RT-PCR followed by Southern blotting (Supplementary Figure S2). To detect LMinduced NK cell responses, $2 \times 10^{5}$ spleen cells were infected with $2 \times 10^{5}$ CFU of LM for $1 \mathrm{~h}$, and subsequently treated with gentamicin $\left(50 \mu \mathrm{g} \mathrm{ml}^{-1}\right)$ until the end of restimulation $(72 \mathrm{~h})$. After $72 \mathrm{~h}$, the frequency of IFN $\gamma$-producing cells was determined by ELISPOT according to standard protocols (BD Biosciences), using an ELISPOT reader (CTL Immunospot S4 analyzer, Cellular Technology Ltd., Cleveland, OH, USA). To determine the CD8 ${ }^{+}$ T-cell or NK-cell component of the responses, spleen cells were depleted of CD8 $\mathrm{T}$ cells using anti-CD8 $\alpha$ (Clone 53-6.7)conjugated magnetic beads, or depleted of NK cells using antiCD49b (Clone DX5)-conjugated magnetic beads according to the manufacturer's instructions (Miltenyi, Auburn, CA, USA).

Assessment of toxicity. Liver toxicity was assessed by visual inspection after killing the mice, and a numerical grade was assigned corresponding to the size and number of visible necrotic plaques. The toxicity was graded as follows: T0 = no lesions (normal appearance); $\mathrm{T} 1=$ uniform light discolouration and firmness; $\mathrm{T} 2=$ white plaques visible covering $\sim 5 \%$ of the liver surface; $\mathrm{T} 3=$ white plaques covering $\sim 10 \%$ of liver surface; $\mathrm{T} 4=$ white plaques covering up to $\sim 30 \%$ of liver surface; and $\mathrm{T} 5=$ white plaques covering $\geqslant 30 \%$ of liver surface. Haematoxylin and eosin (H\&E) staining of thin sections of livers was also done to confirm the presence and extent of hepatic inflammation and necrosis. Briefly, liver tissues were fixed in $10 \%$ formaldehyde for $48 \mathrm{~h}$, and then kept in $70 \%$ ethanol until use. Sections of $1 \mathrm{~mm}$ thickness were stained with $\mathrm{H} \& \mathrm{E}$, and analysed for pathological damage by light microscopy. All pathological analyses were performed by a trained veterinary pathologist. Survival was followed for up to 18 days, and survival curves plotted for the various treatment groups.

Statistical analysis. Effects of treatments on tumour growth, metastasis and immune responses were analysed using the MannWhitney test, Unpaired $t$-test or ANOVA with Sidak post-test for multiple comparisons as indicated. Survival studies were analysed with the Mantel-Cox test. Values of $P<0.05$ were considered statistically significant.

\section{RESULTS}

Efficacy of combination therapy with LM-Mb and $\alpha$ GC in metastatic breast cancer. The therapeutic effect of combining the $\mathrm{LM}-\mathrm{Mb}$ vaccine with $\alpha \mathrm{GC}$ treatment or $\alpha \mathrm{GC}$ physically incorporated into live $\mathrm{LM}-\mathrm{Mb}$ vaccine on metastatic breast cancer was assessed in the 4T1 mouse tumour model. Mice treated with unmodified LM showed marked reduction in metastases when killed at day 18 after initial tumour implantation (Figure 1A), consistent with previous studies showing that this treatment induces a variety of tumoricidal mechanisms (Kim et al, 2009). An improved anti-metastatic effect was observed with LM-Mb compared to LM, suggesting an effect of Mage-b-specific immunity, and a similar level of anti-metastatic activity was observed with administration of $\alpha \mathrm{GC}$ as a single agent. Strikingly, the combination of $\alpha \mathrm{GC}$ with $\mathrm{LM}-\mathrm{Mb}$, either as a separately administered agent (LM-Mb $+\alpha \mathrm{GC}$ ) or by direct incorporation of 

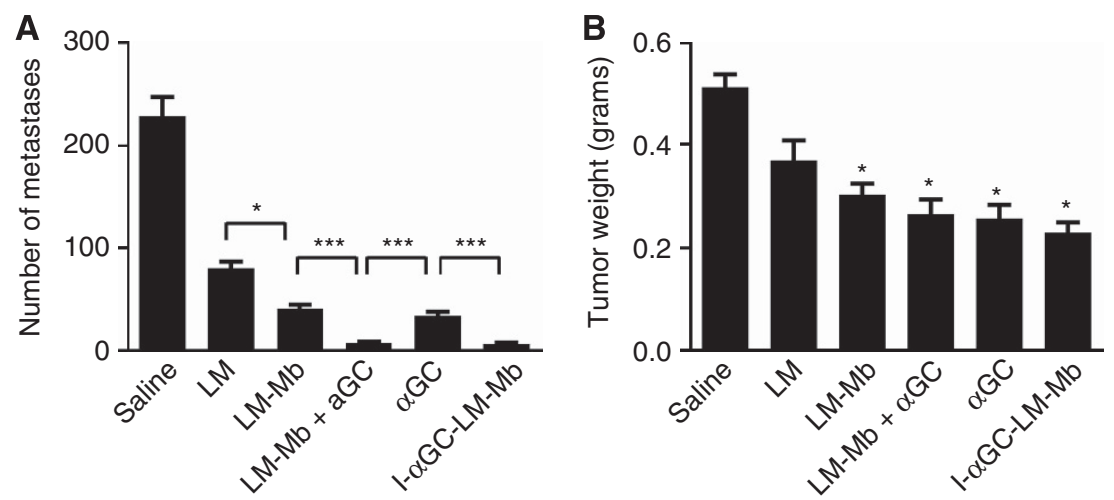

Figure 1. Therapeutic effects of Listeria-based vaccines and $\alpha \mathrm{GC}$ in the $4 \mathrm{~T} 1$ breast cancer model. BALB/c mice were injected with $10^{4} 4 \mathrm{~T} 1$ tumour cells in the mammary fat pad and subsequently treated with various single agents or combination regimens. Single agents were given therapeutically as i.p. injections on days 3, 6 and 9, and consisted of saline (sham treatment), attenuated Listeria monocytogenes (LM), LM expressing Mage-b (LM-Mb) or $\alpha$ GC. Combination regimens consisted of three therapeutic injections of LM-Mb (days 3,6 and 9) followed by three injections of $\alpha \mathrm{GC}$ (days 14, 15 and 16; LM-Mb $+\alpha \mathrm{GC}$ ), or five therapeutic injections of LM-Mb modified by direct incorporation of $\alpha \mathrm{GC}$ (I- $\alpha$ GC-LM-Mb; see Materials and Methods for details). Mice were euthanised on day 18 and analysed for the frequency of metastases (A) and primary tumour weight (B). This experiment was performed three times with consistent results using $N=5$ mice per group, and the results shown average values for means and s.e. for data from all three experiments. For both metastases and tumour weight, all treated groups were significantly different from the saline-treated control groups, with an exception for the tumour weight of the groups treated with $L M$ only. ${ }^{*} P<0.05$, ${ }^{\star \star \star} P<0.001$ (Mann-Whitney test) for comparisons between individual groups as indicated in $\mathbf{A}$, and for comparisons of individual treated groups to saline control group in $\mathbf{B}$.

the glycolipid into the live bacteria (I- $\alpha \mathrm{GC}-\mathrm{LM}-\mathrm{Mb})$, gave a significantly better anti-metastatic effect compared to LM-Mb alone or $\alpha \mathrm{GC}$ alone (Figure 1A). Both of the regimens using combined LM-Mb and $\alpha \mathrm{GC}$ treatment reduced the number of macroscopically visible metastases nearly to zero, with only a rare nodule being detected in these mice. The number of metastases were reduced by I- $\alpha \mathrm{GC}-\mathrm{LM}-\mathrm{Mb}$ in all tissues/organs compared to the saline group (MLN, spleen, kidney, liver and diaphragm), but most dramatically in the MLN (Supplementary Table S1). Significant reductions in the weights of primary tumours were also observed in all treatment groups compared to the saline group (with an exception for LM), although combination treatment did not show significant improvement over single-agent treatment in this parameter (Figure 1B).

Direct incorporation of $\alpha \mathrm{GC}$ into LM-Mb avoids severe toxicity. Although $\alpha \mathrm{GC}$ was equally efficacious in reducing metastases when administered as a free glycolipid following LM$\mathrm{Mb}$ treatment or simultaneously using the direct incorporation approach, analysis of survival of treated mice revealed a striking difference between these two approaches. This was first examined in a short-term survival study in which mice receiving various therapies were observed for 18 days following implantation of 4T1 cells in the mammary fat pad. Whereas all sham-treated 4T1 bearing mice (i.e., saline injections only) survived at least 18 days from the time of tumour initiation, we observed a significant fatality rate starting around day 11 in animals treated with the sequential administration of $\mathrm{LM}-\mathrm{Mb}$ and $\alpha \mathrm{GC}$ as separate agents. In contrast, no deaths were observed over this time period in any of the other treatment groups, including those which received LM$\mathrm{Mb}$ with directly incorporated $\alpha \mathrm{GC}$ (I- $\alpha \mathrm{GC}-\mathrm{LM}-\mathrm{Mb}$; Figure $2 \mathrm{~A}$ ). We then carried out a second more extended survival study to compare time to death in $4 \mathrm{~T} 1$ tumour-bearing mice receiving therapeutic vaccinations with $\mathrm{I}-\alpha \mathrm{GC}-\mathrm{LM}-\mathrm{Mb} v s$ mice receiving only saline injections. Although mice that received only sham immunisations with saline all succumbed by day 20 , mice that received $\mathrm{I}-\alpha \mathrm{GC}-\mathrm{LM}-\mathrm{Mb}$ all survived past day 20 and showed a significant extension (30\%) of overall survival (Figure 2B). This extension of survival was consistent with the marked antimetastatic effect and low toxicity of the I- $\alpha$ GC-LM-Mb treatment.
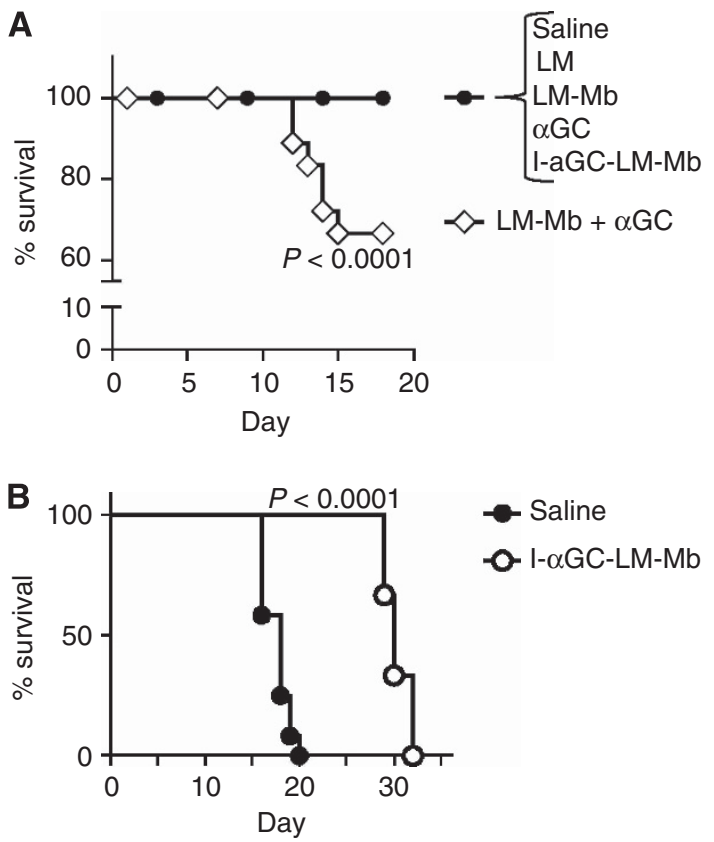

Figure 2. Survival of $4 \mathrm{~T} 1$ tumour-bearing mice treated with various regimens. BALB/c mice were challenged with $4 \mathrm{~T} 1$ tumour cells and immunised therapeutically i.p. with saline or the single or combination regimens as described in Figure 1. The percentage of live animals was determined daily for all groups until all mice were killed on day 18 after tumour challenge (A). This experiment was repeated three times with $N=5$ mice per group, and the results were averaged. Extended survival study for BALB/c mice ( $N=8$ per group) challenged on day 0 with 4T1 tumour cells, and treated therapeutically with i.p. injections of saline alone or $10^{4} \mathrm{CFU}$ of I- $\alpha$ GC-LM-Mb administered every 3 days (B). $P$ values for differences between survival curves are indicated (Mantel-Cox test).

The apparent toxicity leading to accelerate death in animals receiving the separately injected $\mathrm{LM}-\mathrm{Mb}$ and $\alpha \mathrm{GC}$ regimen was investigated by necropsy performed on animals killed at day 18 . 
Focal areas of hepatic necrosis were found and verified by histologic studies of thin sections of the liver (Figure 3A). Such foci were also observed with lower frequency and smaller size in mice that received $\alpha \mathrm{GC}$ alone, but not at all in animals that were treated with I- $\alpha \mathrm{GC}-\mathrm{LM}-\mathrm{Mb}$ or LM-Mb alone (Figure $3 \mathrm{~B}$ ).

Activation of NKT cells in spleens of 4T1 tumour-bearing mice that received I- $\alpha$ GC-LM-Mb. To determine whether LM bacteria modified by direct incorporation of $\alpha$ GC could activate NKT cells in vivo, we assessed the rapid production of IFN $\gamma$ and IL-4, two cytokines that are characteristically for NKT-cell activation. For this purpose we injected naive mice once with LM, $\alpha \mathrm{GC}$ or LM modified by direct incorporation of $\alpha \mathrm{GC}$ (I- $\alpha \mathrm{GC}-\mathrm{LM}$ ) and obtained serum samples at various time points after injection to determine cytokine levels. (The slower adaptive responses to $\mathrm{Mb}$ is irrelevant in this analysis of rapid innate-like NKT-cell responses.) Previous studies have shown that $\alpha \mathrm{GC}$ administered as a free glycolipid induces the production of IFN $\gamma$ and IL- 4 that peak in the serum at $\sim 10-12$ and $2 \mathrm{~h}$, respectively (Venkataswamy et al, 2009). Infection with LM also induces the rapid production of
IFN $\gamma$ in naive mice by multiple cell types, including NK, NKT and $\mathrm{CD}^{+} \mathrm{T}$ cells (North and Conlan, 1998; Brigl et al, 2003; Soudja et al, 2012). We found that I- $\alpha$ GC-LM stimulated a serum IFN $\gamma$ response that was apparent at $10 \mathrm{~h}$, and peaked at $24 \mathrm{~h}$. In contrast, LM infection generated a large transient serum IFN $\gamma$ response, which was first detected at $24 \mathrm{~h}$ (Figure $4 \mathrm{~A}$ ). The accelerated IFN $\gamma$ production seen with I- $\alpha \mathrm{GC}$-LM was consistent with direct NKTcell activation by the $\alpha \mathrm{GC}$ incorporated into the bacteria. Also supporting the conclusion that NKT cells were directly activated, we observed a significant IL-4 response at $2 \mathrm{~h}$ after injection of I- $\alpha$ GC-LM, whereas LM alone did not induce detectable IL-4 (Figure 4B).

To confirm that NKT cells were rapidly activated by treatment with I- $\alpha$ GC-LM-Mb in tumour-bearing mice in vivo, we analysed the percentages of NKT cells in the spleens of mice with 4T1 tumours and metastases that were therapeutically immunised with I- $\alpha$ GC-LM-Mb using CD1d tetramers loaded with $\alpha$ GC. The percentage of NKT cells stained with the tetramer in the spleens of tumour-bearing mice that had received five therapeutic treatments with I- $\alpha$ GC-LM-Mb was either unchanged or slightly increased
A
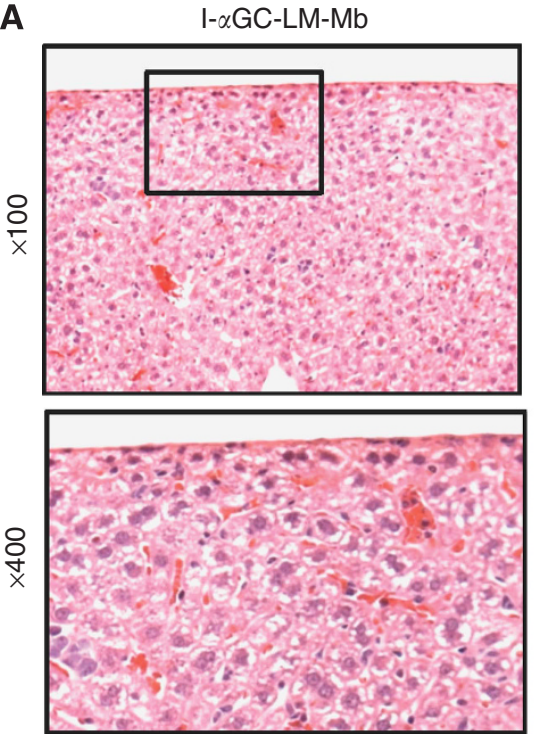
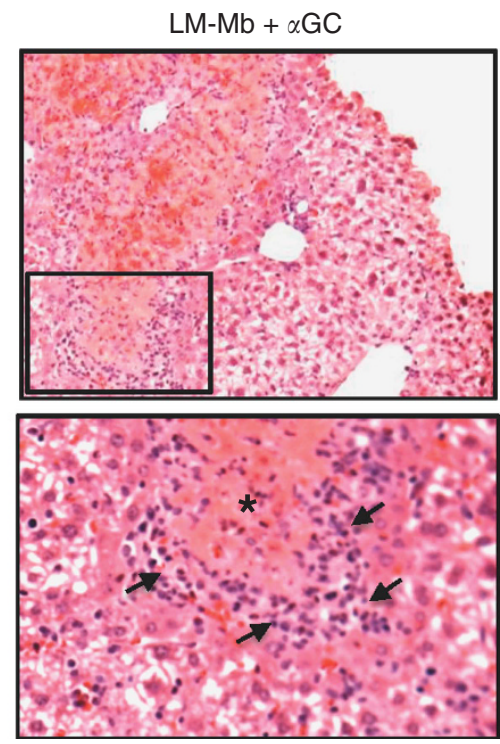

B

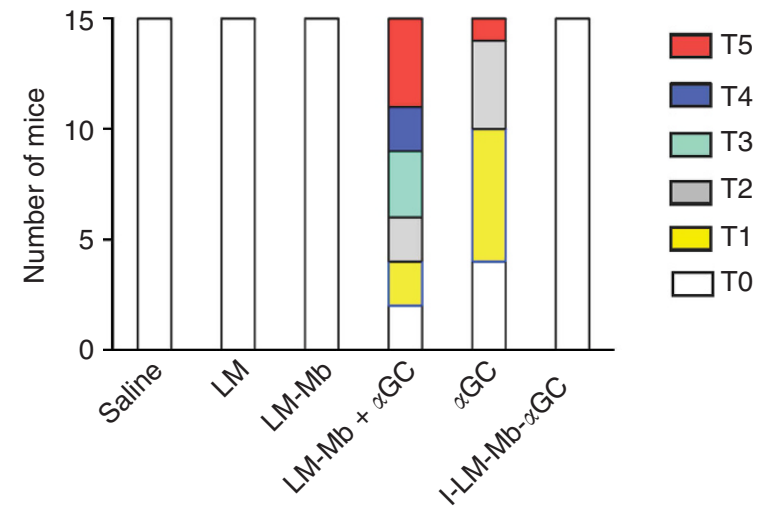

Figure 3. Hepatotoxicity of $\alpha \mathrm{GC}$ administered as a separate agent but not when incorporated into LM-Mb. Representative micrographs of H\&E stained sections from the livers of mice $4 \mathrm{~T} 1$ tumour-bearing mice that received I- $\alpha \mathrm{GC}-\mathrm{LM}-\mathrm{Mb}$ (left) or LM-Mb $+\alpha \mathrm{GC}$ (right) as described in Figure 1, and killed on day 18 (A). Low power views (top) show normal appearance of liver tissue typical for I- $\alpha$ GC-LM-Mb-treated mice, and foci of necrosis and inflammatory cells that were common in mice receiving LM-Mb $+\alpha \mathrm{GC}$. Higher magnification views of the boxed areas are shown in the lower panels, with an area of necrosis marked by the asterisk and arrows indicating leukocyte infiltrates for the LM-Mb $+\alpha G C$ sample. Summary of semiquantitative grading of toxicity based on gross liver pathology (B). BALB/c mice were challenged with 4T1 tumour cells and immunised therapeutically i.p. with saline or the single or combination regimens as described in Figure 1. Toxicity was graded in each mouse by visual inspection of livers at necropsy using a scale from T0 to T5 as described in Materials and Methods. This experiment was repeated three times with $N=5$ mice per group and the results of all experiments are combined in the graph shown. 

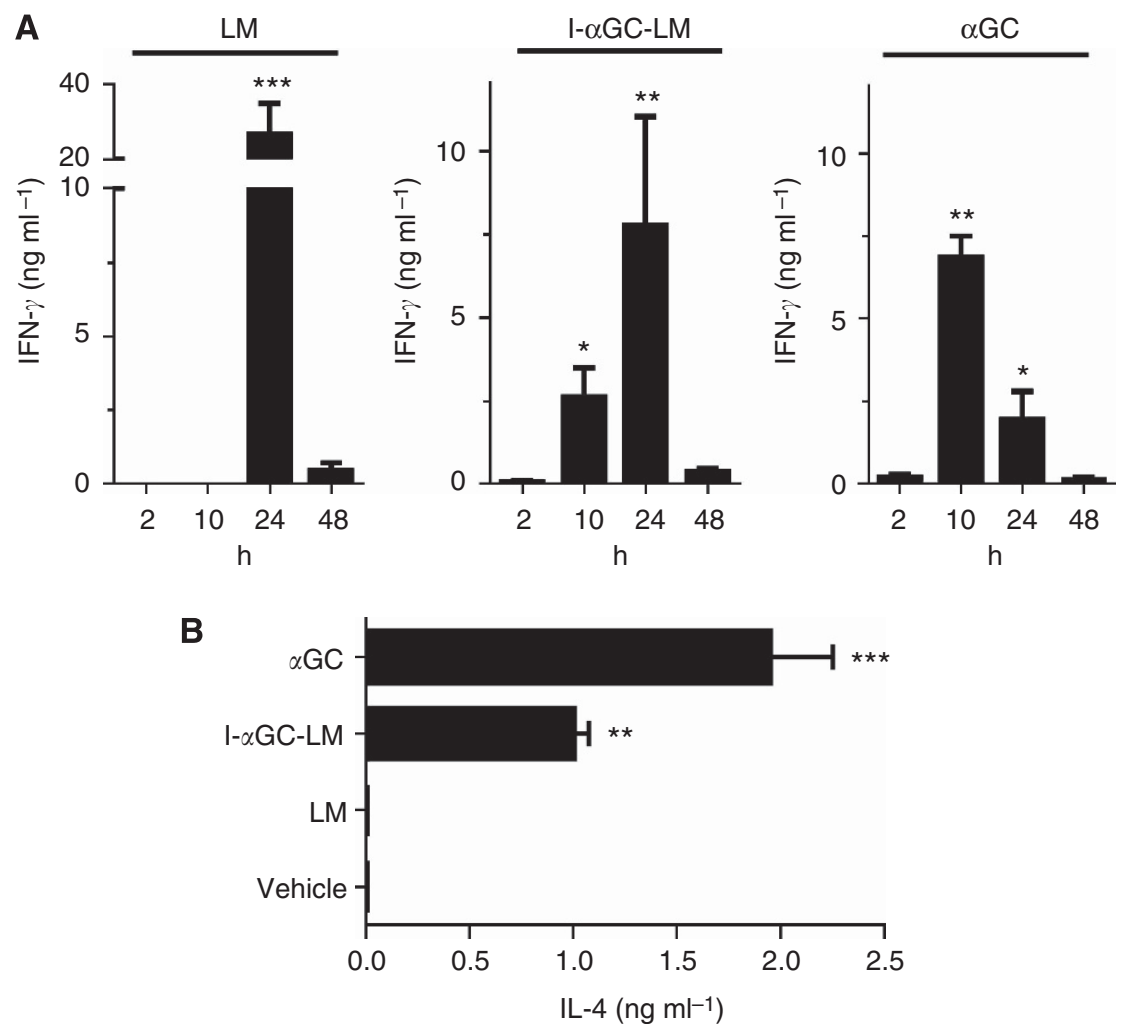

Figure 4. Rapid induction of IFN $\gamma$ and IL-4 by immunisation with I- $\alpha$ GC-LM. BALB/c mice were injected i.p. with inert vehicle, LM, I- $\alpha$ GC-LM or free $\alpha \mathrm{GC}$. Sera were assayed at the indicated times for IFN $\gamma$ by capture ELISA (A). Values of IFN $\gamma$ for vehicle-treated mice were below the limit of detection $\left(<0.1 \mathrm{ng} \mathrm{ml}^{-1}\right.$ ). Sera obtained from mice ( $N=3$ per group) at $2 \mathrm{~h}$ after treatment as in A were assayed for IL-4 by capture ELISA (B). Graphs are a representative of three experiments with $N=3$ mice per group. Mean and s.d. are shown, and significant elevations of IFN $\gamma$ above background levels are indicated by asterisks. ${ }^{\star} P<0.05$, ${ }^{\star \star} P<0.01,{ }^{\star \star \star} P<0.001$; ANOVA with Sidak post-test for multiple comparisons.

compared to saline or LM-treated controls at $0 \mathrm{~h}$ post $\alpha \mathrm{GC}$ injection (Figure 5A and B), while tetramer binding of NKT cells showed a transient decrease in the spleen at $12 \mathrm{~h}$, followed by a rebound to greater than baseline levels at $72 \mathrm{~h}$ (Figure 5A and $\mathrm{B}$ ). This was consistent with the normal activation pattern observed for intact NKT-cell populations in healthy naive mice, which is characterised by rapid TCR downmodulation leading to loss of tetramer staining at earlier time points, followed by TCR reexpression and proliferative expansion of the tetramer staining population by $72 \mathrm{~h}$ (Wilson et al, 2003). In fact, animals that had received prior treatment with either LM-Mb or I- $\alpha \mathrm{GC}-\mathrm{LM}-\mathrm{Mb}$ showed significantly increased expansion of NKT cells at $72 \mathrm{~h}$ after subsequent injection of free $\alpha \mathrm{GC}$, with I- $\alpha \mathrm{GC}$-LM-Mb showing the greater effect (Figure 5A and B). In summary, NKT-cell activation by repeated I- $\alpha \mathrm{GC}-\mathrm{LM}-\mathrm{Mb}$ treatments did not lead to either depletion or anergy of NKT cells in vivo, and may actually have primed NKT cells to respond more vigourously to subsequent stimulation.

Improved $\mathrm{T}$ cell and NK-cell responses in mice vaccinated with I- $\alpha$ GC-LM-Mb. The activation of NKT cells has been frequently shown to lead secondarily to increased CD8 ${ }^{+}$T-cell cross-priming and to NK-cell activation (Hermans et al, 2003; Venkataswamy et al, 2009). Given that NKT-cell activation was augmented by treatment of tumour-bearing mice with I- $\alpha$ GC-LM-Mb, we analysed whether this treatment could also enhance CD8 T cell $\left(\mathrm{CD} 8 \alpha^{+}\right)$and $\mathrm{NK}$-cell $\left(\mathrm{CD} 49 \mathrm{~b} / \mathrm{DX} 5^{+}\right)$responses using the production of IFN $\gamma$ by these cells as an activation marker. First, we analysed antigen-dependent recall responses to Mage-b in the spleens of I- $\alpha$ GC-LM-Mb-treated and control mice upon restimulation with Mage-b in vitro using IFN $\gamma$ ELISPOT assays. This showed significantly higher numbers of IFN $\gamma$ secreting cells in response to stimulation ex vivo by expression of Mage-b by plasmid transfection in the spleens of mice treated with $\mathrm{I}-\alpha \mathrm{GC}$ $\mathrm{LM}-\mathrm{Mb}$ compared to the control mice treated with LM-Mb, $\alpha \mathrm{GC}$ or saline (Figure 6A). These responses were substantially diminished following depletion using anti-CD8 $\alpha$-conjugated magnetic beads. Very low numbers of IFN $\gamma$-producing cells were observed after restimulation by transfecting a plasmid encoding only a control protein, lacZ (Supplementary Figure S3), demonstrating the antigen specificity of the restimulation assay. Although NK cells and a subset of DCs express low levels of CD $8 \alpha$ and can potentially contribute to IFN $\gamma$ responses (Vremec et al, 2007; Shortman and Heath, 2010), the antigen dependence of the observed responses, both at the level of in vivo vaccination and in vitro restimulation, indicated that the responding cells were most likely CD $8 \alpha^{+}$T cells. Similarly, enumeration of CD $8 \alpha^{+}$cells spontaneously producing intracellular IFN $\gamma$ (i.e., as a result of in vivo treatment but without in vitro restimulation) in peripheral blood samples by flow cytometry showed a significantly higher response in mice treated with I- $\alpha$ GC-LM-Mb compared to mice treated with $\mathrm{LM}-\mathrm{Mb}$ or $\alpha \mathrm{GC}$ alone (Figure 6B). In primary tumours, the percentage of $\mathrm{CD} 8 \alpha^{+}$cells producing intracellular IFN $\gamma^{+}$was significantly higher for mice that received I- $\alpha$ GC-LM$\mathrm{Mb}$ compared to mice that received saline, but not compared to mice that received LM-Mb or $\alpha \mathrm{GC}$ alone (Supplementary Figure S4). In summary, these results were consistent with a superior effect of I- $\alpha \mathrm{GC}-\mathrm{LM}-\mathrm{Mb}$ on stimulating the cross-priming of CD $8 \alpha^{+}$T cells specific for TAAs.

We also measured NK-cell $\left(\mathrm{DX}^{+}{ }^{+}\right)$responses in the spleens of I- $\alpha$ GC-LM-Mb-treated and control mice. For this purpose, we infected the spleen cells with LM in vitro and determined the percentage of IFN $\gamma$-producing cells by ELISPOT with and without anti-DX5 magnetic bead depletion. We found that the number of 
A Hours post $\alpha \mathrm{GC}$ injection
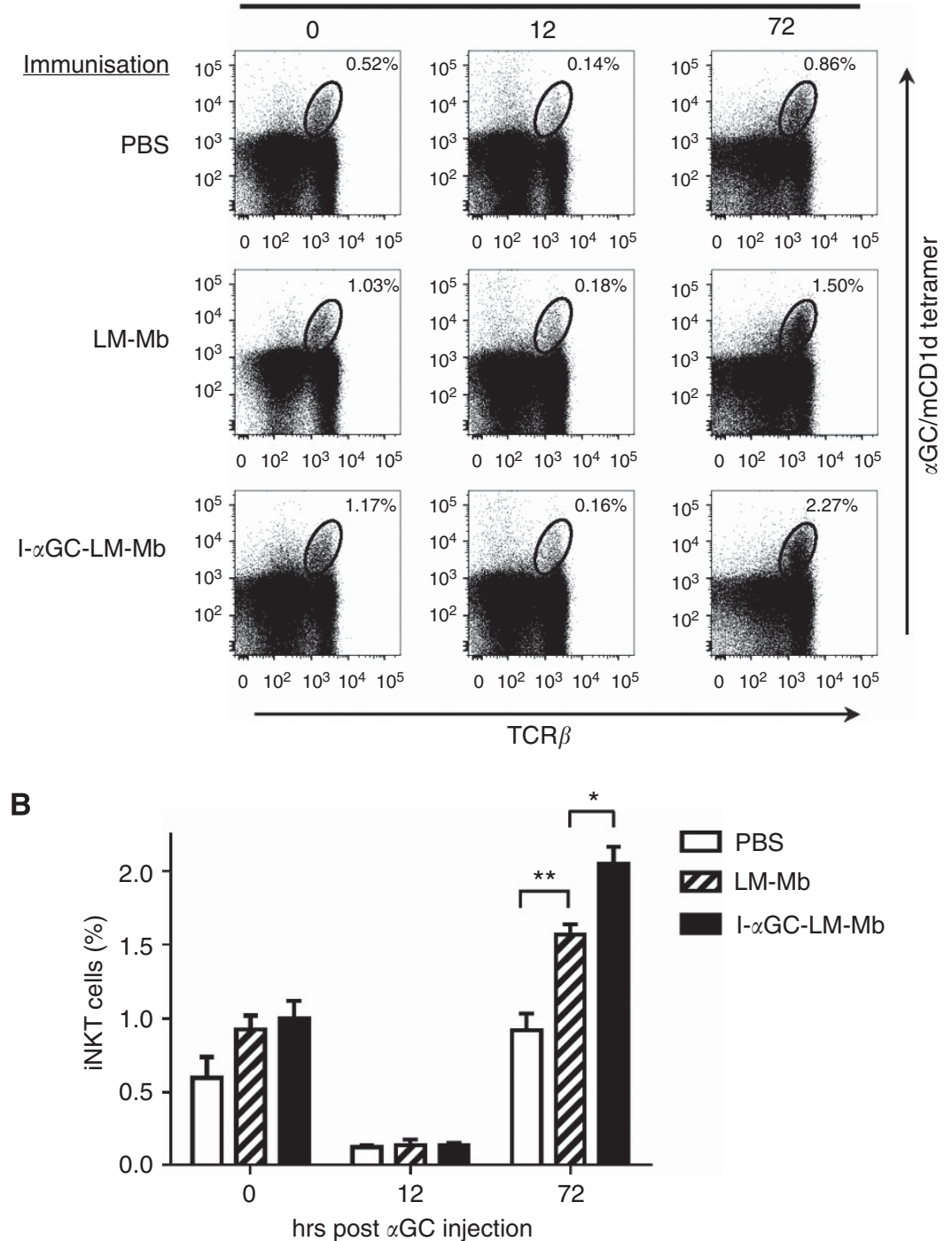

Figure 5. Absence of NKT-cell anergy following repeated therapeutic immunisations with I- $\alpha$ GC-LM. BALB/c mice were challenged with 4 T1 tumour cells and immunised therapeutically with saline, LM-Mb and I- $\alpha$ GC-LM-Mb as described in Figure 1. Fifteen days after the first immunisation, splenocytes were obtained and stained for NKT cells using $\alpha$ GC-loaded CD1d tetramers ( $\mathrm{h}$ time point). In parallel, a group of mice receiving the same treatment was injected i.p. with free $\alpha \mathrm{GC}$ and splenocytes were stained for NKT cells after 12 and $72 \mathrm{~h}$. (A and $\mathbf{B}$ )

Representative dot plots depicting staining with anti-TCR $\beta$ and $\alpha \mathrm{GC}$-loaded CD1d tetramers are shown in $\mathbf{A}$, and the results using $N=3$ mice per group are summarised in $\mathbf{B}$. Mean values and s.e. are shown. ${ }^{\star} P<0.05,{ }^{\star \star} P<0.01$ (ANOVA with Sidak post-test).

cells secreting IFN $\gamma$ in response to restimulation by LM infection was significantly increased in I- $\alpha$ GC-LM-Mb-treated mice compared to mice treated with saline, or with LM-Mb or $\alpha \mathrm{GC}$ alone. These responses were substantially reduced by depletion of DX5 ${ }^{+}$cells, consistent with a large NK-cell component (Figure 6C). Overall, our results indicated that treatment of tumour-bearing mice with combined therapy in the form of $\mathrm{I}-\alpha \mathrm{GC}-\mathrm{LM}-\mathrm{Mb}$ gave superior $\mathrm{CD} 8 \alpha^{+}$T-cell priming against specific TAAs and also enhanced NK cell activation.

Accumulation of I- $\alpha$ GC-LM-Mb in metastases and primary tumours. Previous studies showed enhanced survival and replication of LM in primary and metastatic tumours compared to normal tissues, most likely as a consequence of local immune suppression in the TME (Chandra et al, 2013; Quispe-Tintaya et al, 2013). To assess the impact of $\alpha \mathrm{GC}$ incorporation on survival and replication of I- $\alpha \mathrm{GC}-\mathrm{LM}-\mathrm{Mb}$ in tissues, we analysed bacterial loads in the metastases, tumours, spleens and livers at days 1, 3 and 6 after a single administration of either LM-Mb or I- $\alpha$ GC-LM-Mb $\left(0.5 \times 10^{7}\right.$ CFU; Supplementary Table S2). This showed that both LM-Mb and I- $\alpha$ GC-LM-Mb accumulated and persisted at high levels in primary tumour and metastatic tissues, with higher CFU per gram of tissue than in spleen and liver. Incorporation of $\alpha \mathrm{GC}$ showed modest reductions in CFU in some cases at day 1 (spleen and liver) and day 3 (spleen), but by day 6 these differences were no longer apparent. We thus conclude that incorporation of $\alpha \mathrm{GC}$ into I- $\alpha$ GC-LM-Mb does not significantly diminish its ability to persist and replicate within tumours and metastases.

\section{DISCUSSION}

Although treatment with $\alpha \mathrm{GC}$ as a free glycolipid injection has shown remarkable anti-tumour activity in a variety of mouse models of cancer, phase I clinical trials of this approach in human cancer patients have not shown clear evidence of therapeutic benefit (Schneiders et al, 2011). In the study presented here, we 

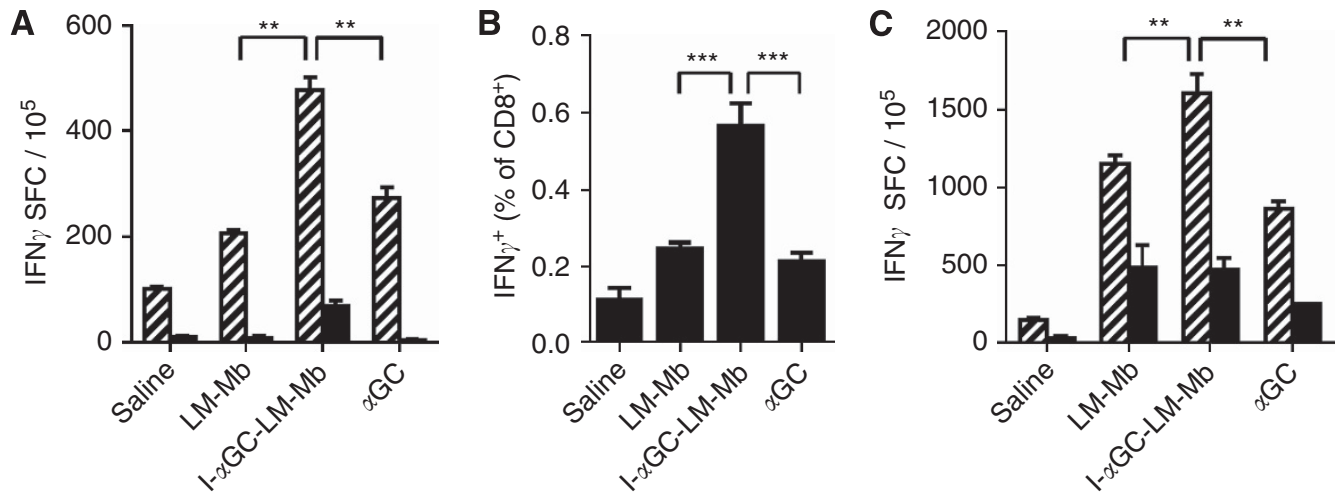

Figure 6. Enhancement of $\mathrm{CD}^{+}$and NK cell responses following therapeutic immunisation with I- $\alpha \mathrm{GC}$-LM-Mb. BALB/c mice were challenged with 4T1 tumour cells and immunised therapeutically i.p. with saline, LM-Mb, I- $\alpha$ GC-LM-Mb or $\alpha G C$ as described in Figure 1. Spleens were collected on day 18 and analysed for IFN $\gamma$-producing cells by ELISPOT. Measurement of IFN $\gamma$ spot-forming cells (SFC) determined by ELISPOT following restimulation in vitro with Mage-b, either without (hatched bars) or with (solid bars) depletion of CD8 $\alpha^{+}$cells (A). White blood cells were analysed directly ex vivo by flow cytometry for intracellular IFN $\gamma^{+}$cells, gating on CD $8 \alpha^{+}$lymphocytes (B). Measurement of IFN $\gamma$ SFC in the spleen was determined by ELISPOT following restimulation in vitro with LM, either without (hatched bars) or with (solid bars) depletion of DX5 $5^{+}$NK cells (C). These experiments were repeated three times with $N=5$ mice per group. Means and s.e. are shown. ${ }^{\star \star} P<0.01,{ }^{\star \star \star} P<0.001$ (Mann-Whitney test).

have explored various therapeutic approaches using $\alpha \mathrm{GC}$ and a LM-based tumour vaccine expressing TAA Mage-b to achieve strong synergistic effects, particularly in the suppression of metastatic disease. In one approach, the LM-Mb vaccine and $\alpha \mathrm{GC}$ were administered as separate components, while an alternate strategy used direct incorporation of $\alpha \mathrm{GC}$ into live $\mathrm{LM}-\mathrm{Mb}$ prior to injection into tumour-bearing animals. This latter approach has been used successfully in previous work to improve the vaccine efficacy of live M. bovis BCG (Venkataswamy et al, 2009). The combination of LM-Mb and $\alpha \mathrm{GC}$, with the glycolipid administered either as a separate series of injections or directly incorporated into the LM-Mb bacteria, was highly effective at reducing the number of metastases, and almost completely eliminated grossly visible metastatic nodules. Most significantly, while the administration of $\mathrm{LM}-\mathrm{Mb}$ and $\alpha \mathrm{GC}$ as separate injections in sequential manner was associated with marked toxicity due to hepatic necrosis, we found that the direct incorporation of the glycolipid into LM-Mb completely eliminated the toxicity while still preserving the marked clinical benefit.

The apparent synergy that we observed between LM and $\alpha \mathrm{GC}$ of inducing anti-tumour immunity without toxicity is likely to be related to the incorporation of $\alpha \mathrm{GC}$ into the LM-Mb. It is likely that the high efficiency of infection of CD $8 \alpha^{+}$DC by LM resulted in enhanced intracellular concentration of $\alpha \mathrm{GC}$ compared to the uptake of free $\alpha \mathrm{GC}$, and may have improved the activation of NKT cells compared to free $\alpha \mathrm{GC}$ in vivo. Indeed, we found that I- $\alpha \mathrm{GC}$ $\mathrm{LM}-\mathrm{Mb}$ significantly increased the population of NKT cells in the spleen compared to free $\alpha \mathrm{GC}$ in $4 \mathrm{~T} 1$ tumour-bearing mice in vivo. Activated NKT cells are known for producing multiple cytokines, such as IL-2, IFN $\gamma$ and IL-4 that can lead to an immunestimulating environment (Bendelac et al, 2007). We found that I- $\alpha$ GC-LM-Mb stimulated iNKT-cell hybridoma cells in vitro, and when injected into mice, I- $\alpha$ GC-LM-Mb rapidly induced elevated levels of IFN $\gamma$ and IL-4 in the serum consistent with direct activation of iNKT cells in vivo.

In addition to directly activating iNKT cells, we also found that repeated immunisations of $4 \mathrm{~T} 1$ tumour-bearing mice with $\mathrm{I}-\alpha \mathrm{GC}$ $\mathrm{LM}-\mathrm{Mb}$ significantly increased the percentage of $\mathrm{CD} 8 \alpha^{+}$cells producing IFN $\gamma$, compared to $\mathrm{LM}-\mathrm{Mb}$ or $\alpha \mathrm{GC}$ alone in vivo. Restimulation in vitro with Mage-b also demonstrated that antigen-specific $\mathrm{CD}^{+}$T-cell responses were significantly enhanced in spleens of mice that received I- $\alpha \mathrm{GC}-\mathrm{LM}-\mathrm{Mb}$ compared to mice that received LM-Mb or free $\alpha \mathrm{GC}$. The $4 \mathrm{~T} 1$ metastases and primary tumour highly express Mage-b (Kim et al, 2008) and are therefore a target for Mage-b-specific $\mathrm{CD} 8^{+} \mathrm{T}$ cells.

It has been shown that LM also infects a variety of tumour cells in vitro and in vivo (Kim et al, 2009). This converts poorly immunogenic tumour cells into sensitised targets for LM-activated CD8 T and NK cells, and partly explains why unmodified LM also has a considerable anti-metastatic effect. Moreover, we found that LM could be used for the selective delivery of anti-cancer agents such as rapidly decaying radioisotopes to the metastases (QuispeTintaya et al, 2013). We demonstrated that LM was rapidly cleared in normal tissues by the immune system but prevented from elimination in the TME by the strong immune suppression. Therefore, it is likely that in the current study I- $\alpha$ GC-LM-Mb has delivered $\alpha \mathrm{GC}$ to metastases as well, and may have attracted NKT cells selectively to these sites. Consistent with this, we found that the number of CFU of I- $\alpha \mathrm{GC}-\mathrm{LM}-\mathrm{Mb}$ per gram of tissue was significantly higher (14- to 34-fold) in the metastases than in the spleens or livers of tumour-bearing mice. In preliminary experiments, we observed that this was correlated with an approximately two- to three-fold enrichment of the frequency of NKT cells in metastases compared to spleen in mice that received I- $\alpha \mathrm{GC}-\mathrm{LM}$ $\mathrm{Mb}$ (unpublished results).

Our results raise the question of why I- $\alpha \mathrm{GC}-\mathrm{LM}-\mathrm{Mb}$ was more effective against the metastases than primary tumours. In a previous study, we found that the percentage of MDSC was much higher in blood (up to $\sim 80 \%$ of circulating leukocytes) than in the primary tumour ( $\sim 6 \%$ of tumour infiltrating leukocytes) in the 4T1 model and in human cancer patients (Diaz-Montero et al, 2009; Chandra et al, 2013). Since metastases of $4 \mathrm{~T} 1$ spread via the blood stream and Listeria infects the MDSC, delivery of I- $\alpha \mathrm{GC}$ $\mathrm{LM}-\mathrm{Mb}$ to the metastases through MDSC may have been more efficient than to the primary tumour. Indeed, the number of CFU of I- $\alpha$ GC-LM-Mb was significantly higher in metastases than primary tumour on day 1 after injection of the bacteria that may have led to a greater accumulation of NKT cells in metastases compared to primary tumours.

In summary, we have demonstrated that a novel combination of a recombinant LM expressing Mage-b modified by direct incorporation of the NKT-cell-activating glycolipid $\alpha \mathrm{GC}$ almost completely eliminated metastases in the $4 \mathrm{~T} 1$ model without apparent toxicity. This correlated with the activation of NKT cells, NK cells and Mage-b-specific CD $8 \alpha^{+} \mathrm{T}$ cells in tumour-bearing mice. The results described here provide a foundation for future 
studies that could combine a method targeting primary tumours, such as surgery or local radiation therapy, with anti-metastatic treatment using I- $\alpha \mathrm{GC}-\mathrm{LM}-\mathrm{Mb}$ as an approach to achieve prolonged remission or cure of aggressive breast cancer, and perhaps of other metastatic cancers.

\section{ACKNOWLEDGEMENTS}

This study was supported by a pilot grant from the Albert Einstein Cancer Center, NCI grant 1R21 CA129470-01, and by RO1 AI45889, and by the Paul F Glenn Center for the Biology of Human Aging Research. Flow cytometry studies were carried out using core facilities supported by the AECC (NIH/NCI CA013330) and the Einstein Center for AIDS Research (NIH AI-51519). LJC is a recipient of the Pew Foundation International Scholars Fellowship Award. We thank Dr Rani Sellers for the useful discussions and pathological examinations.

\section{CONFLICT OF INTEREST}

SAP is a consultant for Vaccinex, Inc. (Rochester, NY), which has licensed technologies for development of NKT-cell-related therapies for cancer and other diseases.

\section{REFERENCES}

Aslakson CJ, Miller FR (1992) Selective events in the metastatic process defined by analysis of the sequential dissemination of subpopulations of a mouse mammary tumor. Cancer Res 52(6): 1399-1405.

Behar SM, Porcelli SA (2007) CD1-restricted T cells in host defense to infectious diseases. Curr Top Microbiol Immunol 314: 215-250.

Bendelac A, Savage PB, Teyton L (2007) The biology of NKT cells. Annu Rev Immunol 25: 297-336.

Brigl M, Bry L, Kent SC, Gumperz JE, Brenner MB (2003) Mechanism of CD1d-restricted natural killer T cell activation during microbial infection. Nat Immunol 4(12): 1230-1237.

Cardoso F, Costa A, Norton L, Cameron D, Cufer T, Fallowfield L, Francis P, Gligorov J, Kyriakides S, Lin N, Pagani O, Senkus E, Thomssen C, Aapro M, Bergh J, Di Leo A, El Saghir N, Ganz PA, Gelmon K, Goldhirsch A, Harbeck N, Houssami N, Hudis C, Kaufman B, Leadbeater M, Mayer M, Rodger A, Rugo H, Sacchini V, Sledge G, van't Veer L, Viale G, Krop I, Winer E (2012) 1st International consensus guidelines for advanced breast cancer (ABC 1). Breast 21(3): 242-252.

Castro F, Leal B, Denny A, Bahar R, Lampkin S, Reddick R, Lu S, Gravekamp C (2009) Vaccination with Mage-b DNA induces CD8 T-cell responses at young but not old age in mice with metastatic breast cancer. Br J Cancer 101(8): 1329-1337.

Chambers RS, Johnston SA (2003) High-level generation of polyclonal antibodies by genetic immunization. Nat Biotechnol 21(9): 1088-1092.

Chandra D, Jahangir A, Quispe-Tintaya W, Einstein MH, Gravekamp C (2013) Myeloid-derived suppressor cells have a central role in attenuated Listeria monocytogenes-based immunotherapy against metastatic breast cancer in young and old mice. Br J Cancer 108(11): 2281-2290.

Diaz-Montero CM, Salem ML, Nishimura MI, Garrett-Mayer E, Cole DJ, Montero AJ (2009) Increased circulating myeloid-derived suppressor cells correlate with clinical cancer stage, metastatic tumor burden, and doxorubicin-cyclophosphamide chemotherapy. Cancer Immunol Immunother 58(1): 49-59.

Gajewski TF, Meng Y, Harlin H (2006) Immune suppression in the tumor microenvironment. J Immunother 29(3): 233-240.

Gonzalez-Angulo AM, Morales-Vasquez F, Hortobagyi GN (2007) Overview of resistance to systemic therapy in patients with breast cancer. $A d v$ Exp Med Biol 608: 1-22.

Gunn GR, Zubair A, Peters C, Pan ZK, Wu TC, Paterson Y (2001) Two Listeria monocytogenes vaccine vectors that express different molecular forms of human papilloma virus-16 (HPV-16) E7 induce qualitatively different $\mathrm{T}$ cell immunity that correlates with their ability to induce regression of established tumors immortalized by HPV-16. J Immunol 167(11): 6471-6479.

Hermans IF, Silk JD, Gileadi U, Salio M, Mathew B, Ritter G, Schmidt R, Harris AL, Old L, Cerundolo V (2003) NKT cells enhance $\mathrm{CD} 4+$ and $\mathrm{CD} 8+\mathrm{T}$ cell responses to soluble antigen in vivo through direct interaction with dendritic cells. J Immunol 171(10): 5140-5147.

Kim SH, Castro F, Gonzalez D, Maciag PC, Paterson Y, Gravekamp C (2008) Mage-b vaccine delivered by recombinant Listeria monocytogenes is highly effective against breast cancer metastases. Br J Cancer 99(5): 741-749.

Kim SH, Castro F, Paterson Y, Gravekamp C (2009) High efficacy of a Listeria-based vaccine against metastatic breast cancer reveals a dual mode of action. Cancer Res 69(14): 5860-5866.

Kruit WH, van Ojik HH, Brichard VG, Escudier B, Dorval T, Dreno B, Patel P, van Baren N, Avril MF, Piperno S, Khammari A, Stas M, Ritter G, Lethé B, Godelaine D, Brasseur F, Zhang Y, van der Bruggen P, Boon T, Eggermont AM, Marchand M (2005) Phase 1/2 study of subcutaneous and intradermal immunization with a recombinant MAGE-3 protein in patients with detectable metastatic melanoma. Int J Cancer 117(4): 596-604.

Marchand M, Punt CJ, Aamdal S, Escudier B, Kruit WH, Keilholz U, Håkansson L, van Baren N, Humblet Y, Mulders P, Avril MF, Eggermont AM, Scheibenbogen C, Uiters J, Wanders J, Delire M, Boon T, Stoter G (2003) Immunisation of metastatic cancer patients with MAGE-3 protein combined with adjuvant SBAS-2: a clinical report. Eur J Cancer 39(1): 70-77.

Metelitsa LS (2011) Anti-tumor potential of type-I NKT cells against CD1d-positive and CD1d-negative tumors in humans. Clin Immunol 140(2): 119-129.

Niethammer AG, Xiang R, Becker JC, Wodrich H, Pertl U, Karsten G, Eliceiri BP, Reisfeld RA (2002) A DNA vaccine against VEGF receptor 2 prevents effective angiogenesis and inhibits tumor growth. Nat Med 8(12): $1369-1375$.

North RJ, Conlan JW (1998) Immunity to Listeria monocytogenes. Chem Immunol 70: 1-20.

Paterson Y, Maciag PC (2005) Listeria-based vaccines for cancer treatment. Curr Opin Mol Ther 7(5): 454-460.

Quispe-Tintaya W, Chandra D, Jahangir A, Harris M, Casadevall A, Dadachova E, Gravekamp C (2013) Nontoxic radioactive Listeria(at) is a highly effective therapy against metastatic pancreatic cancer. Proc Natl Acad Sci USA 110(21): 8668-8673.

Schneiders FL, Scheper RJ, von Blomberg BM, Woltman AM, Janssen HL, van den Eertwegh AJ, Verheul HM, de Gruijl TD, van der Vliet HJ (2011) Clinical experience with alpha-galactosylceramide (KRN7000) in patients with advanced cancer and chronic hepatitis $\mathrm{B} / \mathrm{C}$ infection. Clin Immunol 140(2): 130-141.

Shortman K, Heath WR (2010) The CD8 + dendritic cell subset. Immunol Rev 234(1): 18-31.

Singh R, Dominiecki ME, Jaffee EM, Paterson Y (2005) Fusion to listeriolysin $\mathrm{O}$ and delivery by Listeria monocytogenes enhances the immunogenicity of HER-2/neu and reveals subdominant epitopes in the FVB/N mouse. J Immunol 175(6): 3663-3673.

Soudja SM, Ruiz AL, Marie JC, Lauvau G (2012) Inflammatory monocytes activate memory $\mathrm{CD} 8(+) \mathrm{T}$ and innate NK lymphocytes independent of cognate antigen during microbial pathogen invasion. Immunity 37(3): $549-562$.

Sypniewska RK, Hoflack L, Tarango M, Gauntt S, Leal BZ, Reddick RL, Gravekamp C (2005) Prevention of metastases with a Mage-b DNA vaccine in a mouse breast tumor model: potential for breast cancer therapy. Breast Cancer Res Treat 91(1): 19-28.

Venkataswamy MM, Baena A, Goldberg MF, Bricard G, Im JS, Chan J, Reddington F, Besra GS, Jacobs Jr WR, Porcelli SA (2009) Incorporation of NKT cell-activating glycolipids enhances immunogenicity and vaccine efficacy of Mycobacterium bovis bacillus Calmette-Guerin. J Immunol 183(3): 1644-1656.

Vremec D, O’Keeffe M, Hochrein H, Fuchsberger M, Caminschi I, Lahoud M, Shortman K (2007) Production of interferons by dendritic cells, plasmacytoid cells, natural killer cells, and interferon-producing killer dendritic cells. Blood 109(3): 1165-1173.

Wilson MT, Johansson C, Olivares-Villagomez D, Singh AK, Stanic AK, Wang CR, Joyce S, Wick MJ, Van Kaer L (2003) The response of natural killer $\mathrm{T}$ cells to glycolipid antigens is characterized by surface receptor 
down-modulation and expansion. Proc Natl Acad Sci USA 100(19): 10913-10918.

Yamasaki K, Horiguchi S, Kurosaki M, Kunii N, Nagato K, Hanaoka H, Shimizu N, Ueno N, Yamamoto S, Taniguchi M, Motohashi S, Nakayama T, Okamoto Y (2011) Induction of NKT cell-specific immune responses in cancer tissues after NKT cell-targeted adoptive immunotherapy. Clin Immunol 138(3): 255-265.

Youlden DR, Cramb SM, Dunn NA, Muller JM, Pyke CM, Baade PD (2012) The descriptive epidemiology of female breast cancer: an international comparison of screening, incidence, survival and mortality. Cancer Epidemiol 36(3): 237-248.
Yu KO, Im JS, Molano A, Dutronc Y, Illarionov PA, Forestier C, Fujiwara N, Arias I, Miyake S, Yamamura T, Chang YT, Besra GS, Porcelli SA (2005) Modulation of CD1d-restricted NKT cell responses by using $\mathrm{N}$-acyl variants of alpha-galactosylceramides. Proc Natl Acad Sci USA 102(9): 3383-3388.

This work is published under the standard license to publish agreement. After 12 months the work will become freely available and the license terms will switch to a Creative Commons AttributionNonCommercial-Share Alike 3.0 Unported License.

Supplementary Information accompanies this paper on British Journal of Cancer website (http://www.nature.com/bjc) 